Article

\title{
The Absence of Pyruvate Kinase Affects Glucose-Dependent Carbon Catabolite Repression in Bacillus subtilis
}

\author{
Joana Sousa ${ }^{1,2}$, Philipp Westhoff ${ }^{1,3}$, Karen Methling ${ }^{1}$ and Michael Lalk ${ }^{1, *(1)}$ \\ 1 Institute of Biochemistry, University of Greifswald, 17487 Greifswald, Germany; joanasousa100@gmail.com (J.S.); \\ pd.westhoff@gmail.com (P.W.); methling@uni-greifswald.de (K.M.) \\ 2 Innovayt S/A, Av. João Paulo II 30, 4715-213 Braga, Portugal \\ 3 Institute of Plant Biochemistry, Heinrich-Heine-Universität Düsseldorf, Universitätsstraße 1, \\ 02104 Düsseldorf, Germany \\ * Correspondence: lalk@uni-greifswald.de; Tel.: +49-3834-420-4867
}

Received: 25 August 2019; Accepted: 30 September 2019; Published: 4 October 2019

check for updates

\begin{abstract}
Pyruvate is a key intermediate of diverse metabolic pathways of central carbon metabolism. In addition to being the end product of glycolysis, pyruvate is an essential carbon distribution point to oxidative metabolism, amino acid and fatty acid syntheses, and overflow metabolite production. Hence, a tight regulation of pyruvate kinase (Pyk) activity is of great importance. This study aimed to analyze targeted metabolites from several pathways and possible changes in Bacillus subtilis lacking Pyk. Wild type and $\Delta$ pyk cells were cultivated in chemically defined medium with glucose and pyruvate as carbon sources, and the extracted metabolites were analyzed by ${ }^{1} \mathrm{H}-\mathrm{NMR}$, GC-MS, HPLC-MS, and LC-MS/MS. The results showed that the perturbation created in the pyruvate node drove an adaptation to new conditions by altering the nutritional compounds' consumption. In $\Delta$ pyk, pyruvate, which is subject to glucose-dependent carbon catabolite repression, did not comply with the hierarchy in carbon source utilization. Other metabolic alterations were observed such as the higher secretion of the overflow metabolites acetoin and 2,3-butanediol by $\Delta$ pyk. Our results help to elucidate the regulatory transport of glucose and pyruvate in B. subtilis and possible metabolic reroute to alternative pathways in the absence of Pyk.
\end{abstract}

Keywords: Bacillus subtilis; metabolomics; pyruvate kinase; pyruvate; carbon catabolite repression; overflow metabolites

\section{Introduction}

The bacterium Bacillus subtilis is ubiquitous in nature, living primarily in the soil and associated with water sources, and can successfully adapt to changes in the environment [1,2]. Being the best characterized member of Gram-positive bacteria, B. subtilis is currently the focus of interest in biotechnology and industry due to its easy genetic manipulation, the availability of extensive physiological and biochemical data, and due to being an efficient producer of economically valuable metabolites $[1,3,4]$. In this context, knowledge of metabolomic flux pathways and, consequently, improved strategies for the overproduction of desired metabolites have made B. subtilis a potent applicant organism in a wide industrial field. Pyruvate is a key intermediate of various central carbon metabolism pathways, acting as a branching point of glycolysis and tricarboxylic cycle (TCA cycle), and a substrate of fatty acid and amino acid synthesis. The metabolic link between the glycolysis and the TCA cycle is represented by the phosphoenolpyruvate (PEP)-pyruvate-oxaloacetate node, 
which directs carbon flux and acts as a switch point for carbon flux distribution within the central metabolism [5]. Furthermore, poor coordination between glucose consumption and precursor synthesis in the TCA cycle has been seen as one of the responsible causes for the incomplete oxidation of glucose and production of alternative metabolites-namely, overflow metabolites [6-8]. Therefore, the control of pyruvate homeostasis and its fate is of great importance for cell robustness and viability during environmental changes.

It is well known that in B. subtilis, preferred carbon sources, such as glucose, are able to repress the transport and catabolism of alternative substrates. This carbon catabolite repression (CCR) is achieved through the transcriptional control of genes required for the utilization of secondary carbon sources, imposing a hierarchy in the use of the available nutrients and preventing the waste of resources. Different mechanisms exist in bacteria for the signal transduction pathways that lead to the CCR. In B. subtilis, the CCR is mediated by the phosphotransferase system (PTS). PTS is a multiprotein phosphorelay mechanism that catalyzes the phosphorylation of incoming sugar substrates and their simultaneous translocation across the cell membrane. The CCR is also mediated by the catabolite control protein $\mathrm{A}(\mathrm{CcpA})$, a pleiotropic transcription factor and a master regulatory protein, which can also function as a carbon catabolite activator (CCA) in certain genes, the histidine-containing protein HPr of the PTS, and the bifunctional HPr kinase/phosphorylase (HPrK) [9-13].

During sugar uptake, a chain reaction is triggered inside the cells, where, after successive phosphorylation, a phosphoryl group is transferred to the His15 residue of the HPr. Ultimately, the HPr-His-P transfers the phosphoryl group to the enzymatic transporter EII, allowing the uptake of glucose. Furthermore, it is known that when the intracellular concentration of fructose bisphosphate $(\mathrm{FbP})$ is high, HPr can also be phosphorylated at Ser46 by the HPr kinase/phosphorylase (HPrK). This reaction allows the binding of HPr-Ser-P to CcpA. The HPr-Ser-P-CcpA interaction permits the binding of CcpA to specific sites on the DNA and, thereby, represses or activates the transcription of determined genes $[10,11]$.

Although extensive studies have been carried out concerning the CCR phenomenon, the characterization of $B$. subtilis cultivated in pyruvate and carbon source co-utilization studies using pyruvate are still scarce.

Recently, a pyruvate transport system was identified and characterized in $B$. subtilis $[8,14,15]$. Charbonnier and co-workers verified that the preferred carbon sources in B. subtilis, glucose or malate, trigger the binding of CcpA upstream of the $p f t A B$ gene, which encodes a pyruvate facilitated transporter, resulting in the repression of pyruvate utilization. When these preferred carbon sources are absent, CcpA repression is diminished [8]. Furthermore, a two-component regulatory system named LytST is also involved in pyruvate metabolism and uptake through the membrane. LytST induces the expression of $p f t A B$ in the presence of external pyruvate. However, when the pyruvate flux into the cells is high, $p f t A B$ transcription is retro-inhibited via LytST. It was also shown that LytST activity works in a pyruvate dose manner, which permits the balance of intracellular pyruvate levels and adaptation to environmental changes [8]. The ability to use diverse carbon sources represents a key adaptation mechanism that allows cells to thrive in their natural habitat. Building on this knowledge, we became interested in how pyruvate is metabolized. Specifically, these observations raise the question of whether a co-assimilation of pyruvate and the preferred carbon source, glucose, from the medium would change in the presence of a high pyruvate gradient. So far, little is known concerning the absence of Pyk, how this affects the uptake and secretion patterns of pyruvate and other carbon sources in B. subtilis. Thus, we wanted to provide deeper insights into the physiology and metabolic responses of $B$. subtilis 
to the absence of pyruvate kinase (Pyk), and, ultimately, give hints towards the possible rerouting of carbon fluxes through alternative pathways. To do so, a combination of ${ }^{1} \mathrm{H}-\mathrm{NMR}$ spectroscopy as well as chromatographic separation coupled with mass spectrometry (GC-MS, HPLC-MS, and LC-MS/MS) was applied, allowing the identification of the extra and intracellular metabolites of wild type (wt) and $\Delta$ pyk cells.

\section{Results}

2.1. Concomitant Consumption of Glucose and Pyruvate by $\Delta$ Pyk-Carbon Catabolite Repression of Pyruvate Uptake in the Presence of Glucose Was Relieved

For the analysis of B. subtilis wt and $\Delta$ pyk, cells were cultivated in a chemically defined medium with a mixture of glucose and pyruvate (M9GlcPyr) as carbon sources. The growth curves arising from this condition are represented in Figure 1 (black lines).
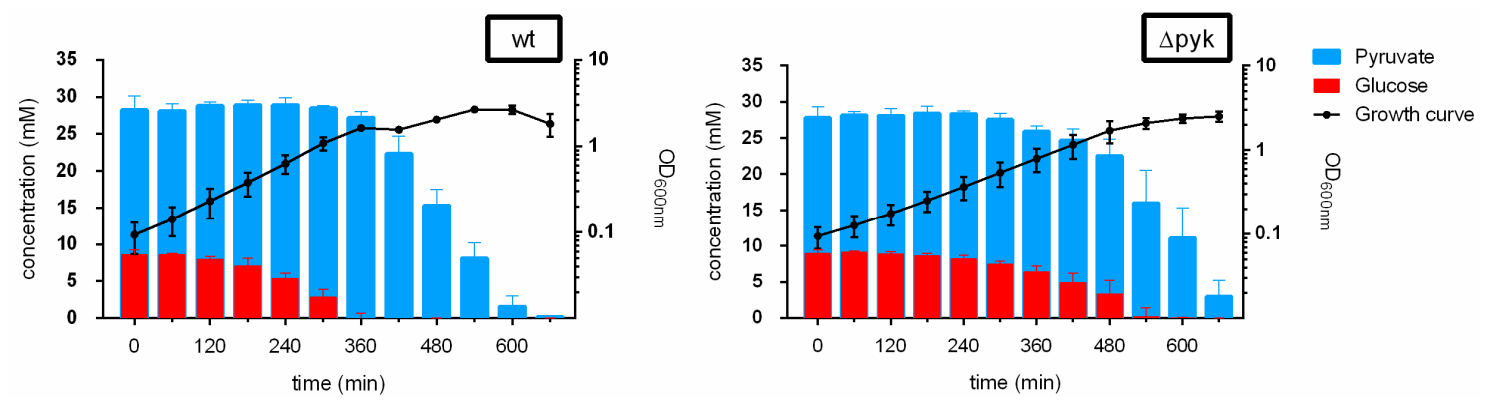

Figure 1. Growth curves and extracellular concentrations of glucose and pyruvate of Bacillus subtilis wild type (wt) and pyruvate kinase ( $\Delta$ pyk) under M9GlcPyr medium cultivation. Black lines illustrate the growth curves, while colored columns represent the external concentration of glucose (red) and pyruvate (blue) at each hour. Data are represented as the mean values \pm standard deviation (SD) of 4 biological replicates. M9GlcPyr-a mixture of glucose and pyruvate.

Pyk mutant cells showed a growth delay during the exponential phase when compared to wt, even though both strains reached the same maximum optical density (OD) (OD 2.5) (Table S1). For wt, a diauxic growth curve was visible with an intermediate stationary phase between 360 and $420 \mathrm{~min}$ at OD 1.6.

Using Nuclear Magnetic Resonance (NMR) spectroscopy technique, changes in the concentration of extracellular metabolites were monitored in a time-dependent manner (Figure 2).

These results revealed that, in the $\mathrm{wt}$, pyruvate consumption was initiated at $360 \mathrm{~min}$ of cultivation, when glucose was depleted from the medium (Figures 1 and 2, and Table 1). On the contrary, pyruvate uptake in $\Delta$ pyk started at $360 \mathrm{~min}$, when $6.4 \pm 0.8 \mathrm{mM}$ of glucose was still available, showing a concomitant consumption of both carbon sources.

Interestingly, acetate was accumulated in similar concentrations in both strains and no uptake was detected until the end of cultivation (Figure 2).

Other typical overflow metabolites produced by B. subtilis, such as acetoin and 2,3-butanediol, were detected in both cultivations. However, these were secreted in notably higher amounts in $\Delta$ pyk, than in the wt (Table S2). In both strains, these metabolites were taken up when external glucose was exhausted. The wt secreted significantly larger amounts of 2-oxoglutarate, valine, and branched-chain amino acid (BCAA) degradation products such as 2-ketoisovalerate, isobutyrate, 2-methylbutyrate, and isovalerate. Moreover, the concentration of valine and 2-ketoisovalerate started to drop when glucose was completely taken up in both strains. 


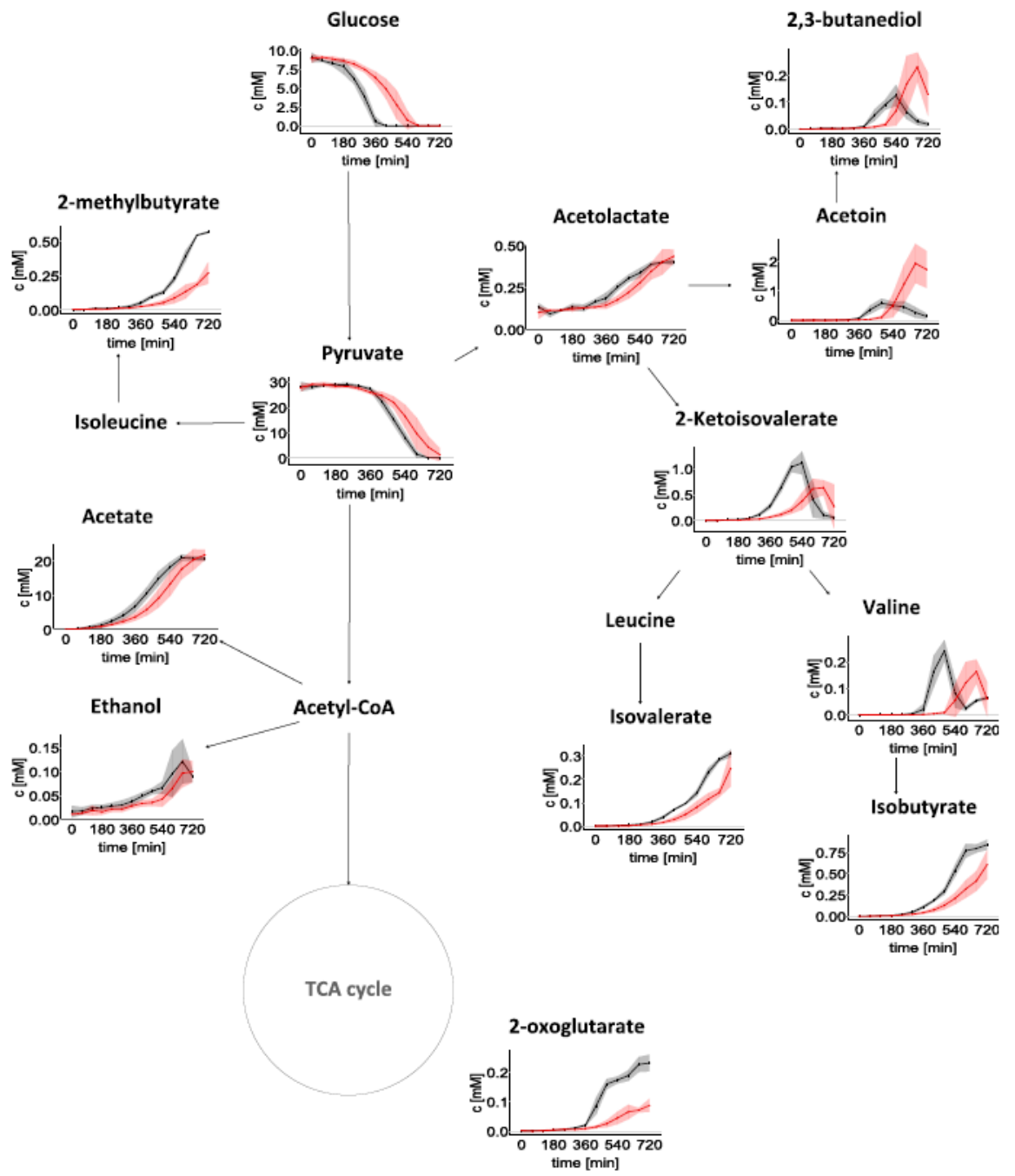

\section{wt - M9GlcPyr \\ pyk mutant - M9GIcPyr}

Figure 2. Time-resolved extracellular metabolites under M9GlcPyr medium cultivation. Absolute concentrations of consumed and secreted metabolites by wt (black) and $\Delta$ pyk (red) are displayed. Data are represented as the mean concentrations $\pm \mathrm{SD}$ (shaded) of quadruplicate samples.

Table 1. Glucose and pyruvate concentrations $(\mathrm{mM})$ present in the supernatant during the time cultivation of wt and $\Delta$ pyk. Data are shown as the mean values \pm SD of quadruplicate samples.

\begin{tabular}{ccccccccc}
\hline & \multicolumn{3}{c}{ wt } & \multicolumn{3}{c}{$\Delta$ pyk } \\
\cline { 2 - 8 } $\begin{array}{c}\text { Time } \\
(\mathbf{m i n})\end{array}$ & Glucose & \multicolumn{3}{c}{ Pyruvate } & Glucose & Pyruvate \\
\cline { 2 - 9 } & $\begin{array}{c}\text { Concentration } \\
(\mathbf{m M})\end{array}$ & SD & $\begin{array}{c}\text { Concentration } \\
(\mathbf{m M})\end{array}$ & SD & $\begin{array}{c}\text { Concentration } \\
(\mathbf{m M})\end{array}$ & SD & $\begin{array}{c}\text { Concentration } \\
(\mathbf{m M})\end{array}$ & SD \\
\hline 0 & 8.59 & 0.65 & 28.25 & 1.85 & 8.93 & 0.53 & 27.80 & 1.52 \\
60 & 8.62 & 0.17 & 28.10 & 0.98 & 9.09 & 0.20 & 28.16 & 0.48 \\
120 & 7.98 & 0.38 & 28.80 & 0.49 & 8.88 & 0.33 & 28.10 & 0.93 \\
180 & 7.05 & 1.04 & 28.93 & 0.62 & 8.63 & 0.31 & 28.41 & 0.99 \\
240 & 5.31 & 0.73 & 28.91 & 0.98 & 8.20 & 0.48 & 28.33 & 0.44 \\
300 & 2.82 & 1.09 & 28.49 & 0.38 & 7.49 & 0.41 & 27.55 & 0.82 \\
360 & 0.00 & 0.68 & 27.24 & 0.81 & 6.41 & 0.80 & 25.94 & 0.73 \\
420 & 0.00 & 0.00 & 22.34 & 2.35 & 4.90 & 1.33 & 24.69 & 1.55 \\
480 & 0.00 & 0.04 & 15.32 & 2.24 & 3.35 & 1.91 & 22.53 & 2.35 \\
540 & 0.01 & 0.01 & 8.14 & 2.10 & 0.20 & 1.20 & 16.03 & 4.51 \\
600 & 0.00 & 0.02 & 1.59 & 1.39 & 0.05 & 0.04 & 11.14 & 4.20 \\
660 & 0.02 & 0.02 & 0.20 & 0.04 & 0.03 & 0.03 & 2.99 & 2.25 \\
\hline
\end{tabular}




\subsection{Intracellular Metabolome Revealed Several Central Metabolic Pathways Altered in $\triangle P y k$}

For the investigation of possible metabolic changes in B. subtilis lacking Pyk, the intracellular metabolome was also monitored under the same growing conditions. During the exponential growing phase, wt and $\Delta$ pyk cells were harvested and quadruplicate samples were inspected using GC-MS, LC-MS, and LC-MS/MS.

Altogether, 92 metabolites from several metabolic pathways were identified and relatively quantified (Table S3). Several metabolites showed altered concentration levels when both strains were compared. The major differences were the notably increased levels of glycolytic metabolites in $\Delta \mathrm{pyk}$, such as phosphoenolpyruvate (PEP), with a fold change (FC) of 10, followed by glucose 6-phosphate (FC 5.0), fructose 6-phosphate (FC 4.6), 3-phosphoglycerate (FC 3.1), and 2-phosphoglycerate (FC 3.2) (Figure 3).

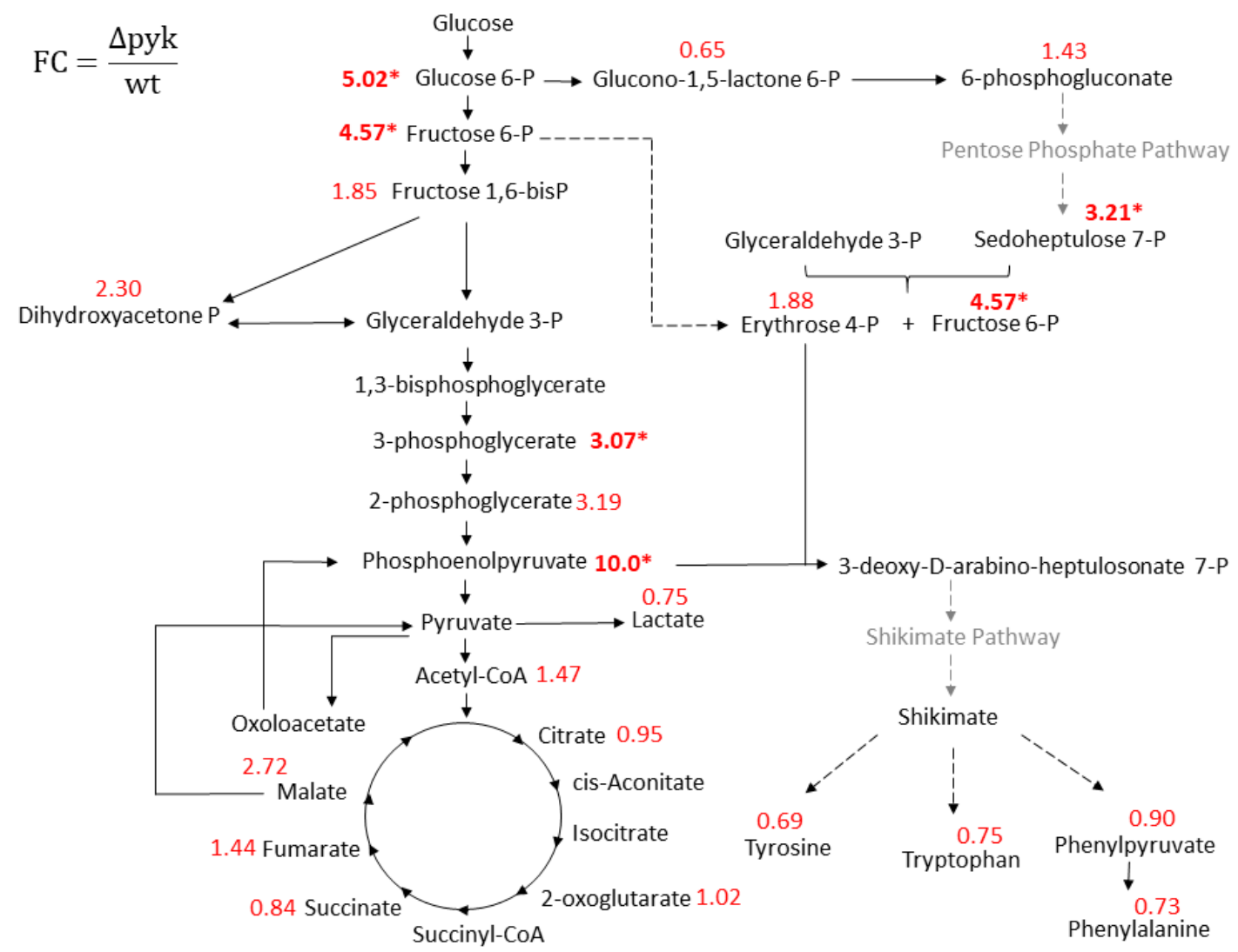

Figure 3. Fold change (FC) of some intracellular metabolites from central metabolism. Relative levels of metabolites from central metabolism for $\Delta$ pyk as compared with wt under M9GlcPyr cultivation. FC was determined using the relative quantification of four biological replicates. Significant alterations $(p$-value $\leq 0.05)$ are marked in bold and asterisk.

Considering the Pentose Phosphate Pathway (PPP), sedoheptulose 7-phosphate presented significant ( $p$-value $\leq 0.05$ ) altered concentration levels (FC 3.2). In the TCA cycle, although 2-oxoglutarate secretion during late exponential and stationary phase was lower in $\triangle \mathrm{pyk}$, the intracellular concentration at 0.5 OD—when intracellular sampling was conducted—was similar in both strains (FC 1.0).

Among the nucleotides, nucleoside triphosphate uridine triphosphate (UTP) and cytidine triphosphate (CTP) were determined in significantly lower amounts in $\triangle$ pyk (FC 0.40 and 0.38 , respectively), as well as inosine monophosphate (IMP) (FC 0.40) and the deoxy nucleotides deoxycytidine triphosphate (dCTP (FC 0.27) and deoxythymidine monophosphate (dTMP) (FC 0.47). On the contrary, adenosine-monophosphate (AMP) was the only nucleotide with statistically higher levels in $\Delta$ pyk (FC 2.1) (Figure 4 and Table S3). 


\section{A}

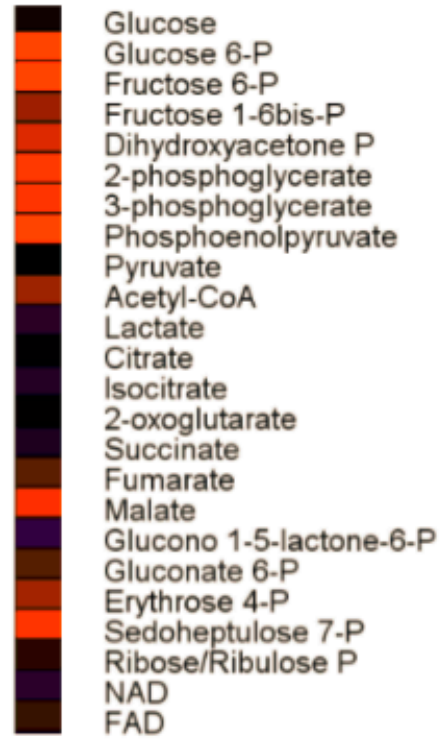

B

\begin{tabular}{|l}
\hline 4-hydroxy-L-proline \\
\hline 5-oxoproline \\
Asparagine \\
Aspartate \\
Alanine \\
Glycine \\
Isoleucine \\
Leucine \\
Lysine \\
Methionine \\
Ornithine \\
Phenylalanine \\
Proline \\
Serine \\
Threonine \\
Tryptophan \\
Tyrosine \\
Urea \\
Valine \\
Glutamate \\
Glutamine \\
Histidine \\
Cysteine \\
Phenylpyruvate
\end{tabular}

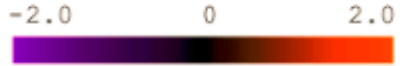

$$
\log _{2} \frac{\Delta \text { pyk }}{w t}
$$

\section{C}

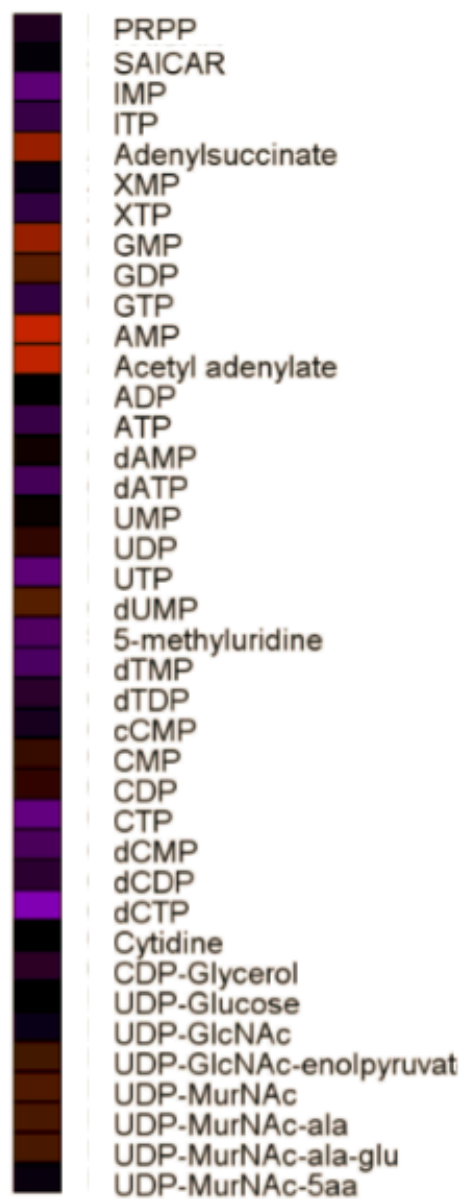

Figure 4. Heat map of intracellular metabolite levels of glycolysis, tricarboxylic (TCA) cycle and Pentose Phosphate Pathway (PPP) (A), amino acids (B), and intermediates of purine and pyrimidine metabolism and cell wall precursors (C), in wt and $\Delta$ pyk under M9GlcPyr cultivation. The color code represents the $\log _{2} \mathrm{FC}$ between $\Delta \mathrm{pyk}$ and wt, whereas increased levels are indicated in orange and lower levels in purple. Data are shown as the mean of four biological replicates. P: phosphate.

\section{Discussion}

\subsection{Carbon Catabolite Repression of Pyruvate Uptake in $\Delta$ Pyk Cells Was Relieved}

The present study comprehensively monitored changes in the intra and extracellular metabolites of B. subtilis but also the alterations in the metabolite uptake and secretion triggered by the absence of Pyk. An interesting outcome of this study is that pyruvate utilization was subject to glucose-dependent CCR in wt cells, while this repression was not observed in $\Delta$ pyk. The rise in pyruvate influx in wt cells initiated at $360 \mathrm{~min}$ of growth, only after external glucose was depleted, indicating that pyruvate was susceptible to glucose-dependent CCR. This phenomenon is also perceived by the diauxic growth 
curve obtained, not described so far. On the other hand, in $\Delta p y k, 6.4 \pm 0.8 \mathrm{mM}$ of glucose was still available in the medium when pyruvate consumption was rapidly initiated. The CCR derepression seen in $\Delta$ pyk can be hypothesized as a cellular metabolic response for the immediate need of other carbon sources, since the central metabolic pathway is possibly altered due to the accumulation of glycolytic metabolites (discussed below).

A pyruvate transport mechanism regulated by the master regulator CcpA was recently discovered. The CcpA controls the expression of $p f t A B$ that encodes a pyruvate facilitated transporter in accordance with the absence/presence of glucose or malate. With the knockout and overexpression of $p f t A B$, it was concluded that the pyruvate gradient observed between the outside and the inside of the cell drives the facilitated PftAB-mediated transport of pyruvate across the cell membrane [8]. In our study, when $\Delta$ pyk cells are growing in M9GlcPyr and the intracellular pyruvate pool is presumably low, LytST might respond by inducing $p f t A B$ transcription sense due to the high pyruvate gradient concentration, leading to a rapid increase in pyruvate influx. In wt, where the pyruvate concentration is under physiological levels inside the cells and the pyruvate gradient is lower, LytST induction is probably impaired and/or the repression of $p f t A B$ by $C$ cpA is highly active. When glucose is completely consumed, the CCR effect on $p f t A B$ is relieved and wt cells are able to take up pyruvate as a nutrient source.

Preliminary studies were also conducted under cultivation with glucose as the single carbon source (data not shown). In these, the deletion of pyk resulted in the impairment of cell growth, a slow consumption of glucose from medium, and an accumulation of intracellular FbP (FC 5.3), when compared to the wt. It is speculated that a possible cause for the low glucose consumption rate seen in $\Delta$ pyk is the impairment of glucose uptake by the PTS system due to the accumulation of high levels of FbP. The concentration of FbP has been proposed to regulate the PTS system at the level of EII-mediated uptake. A high concentration of FbP directly stimulates the phosphorylation of HPr through HPrK, favoring the formation of the HPr-Ser-P-CcpA complex [16,17]. Further experiments are necessary to clarify the glucose dynamics in $\triangle$ pyk and the possibility of the PEP-dependent phosphotransferase system being impaired. These should include the inspection of other PTS sugar transport by $\Delta$ pyk, the quantization of HPr-Ser-P, and the HPrK activity levels.

\subsection{The TCA cycle and the Pentose Phosphate Pathway (PPP) Are Possibly Altered in $\triangle$ pyk}

In the initial exponential growth of wt cells with available glucose and pyruvate, no TCA cycle metabolites were detected outside the cells, which is predicted to be weakly active during this growth phase [18]. In B. subtilis, when preferred carbon sources are available in the medium, the activity of the first TCA cycle enzymes is inhibited by several regulatory proteins [10,19]. When the external glucose was depleted, an efflux of 2-oxoglutarate in wt was initiated (Figure 2), suggesting an increase in TCA cycle activity in response to cell requirements. At this time point (360 min), pyruvate consumption also begun, which can be used for replenishment of the TCA cycle.

As previously discussed, during the late exponential growth phase of $\Delta$ pyk, pyruvate uptake is initiated when glucose is still available. Notably, at this time, 2-oxoglutarate secretion begins, reaching $30 \%$ of the concentration seen in wt $(0.23 \pm 0.03 \mathrm{mM}$ and $0.07 \pm 0.01 \mathrm{mM}$ for wt and $\Delta$ pyk, respectively) at the end of cultivation. Nevertheless, in stationary phase, TCA cycle repression was possibly relieved for the use of alternative carbon sources in the central metabolic pathways. Thus, pyruvate that is crossing into the cell is oxidized to acetyl-CoA production or catabolized by pyruvate carboxylase to oxaloacetate, and immediately directed to the TCA cycle.

As previously described, the growth of $\Delta$ pyk resulted in accumulation of glucose 6-phosphate. It has been reported that the lack of Pyk in Escherichia coli and B. subtilis under glucose medium increases the flux of glucose 6-phosphate through the PPP [20-23]. Although just a few metabolites of this pathway were identified by our method, the results are consistent with this finding, since sedoheptulose 7-phosphate showed higher amounts in $\Delta$ pyk. The perturbation seen in glycolysis in the mutant could have caused the reroute of flux metabolism to compensate the required amount of $\mathrm{NAD}(\mathrm{P}) \mathrm{H}$ and synthesis of nucleotide precursors [24,25]. 


\subsection{Overflow Metabolites: Increased Secretion of Acetoin and 2,3-Butanediol by $\Delta p y k$}

Common phenomena during B. subtilis cultivation with high availability of carbon sources are the production and secretion of overflow metabolites, even in aerobic conditions. Studies suggest that the onset of overflow metabolism during a surplus of carbon and energy is driven by the saturation of the electron transport chain that generates the demanding ATP in fast-growing cells, and the imbalance of the intracellular NADH/NAD ${ }^{+}$ratio observed prior to the beginning of fermentation [26,27].

As typical in B. subtilis, acetate was the overflow compound with the highest secretion amount [24]. The expression of the genes involved in the synthesis of acetate is activated by CcpA in the presence of glucose (CCA effect). Moreover, the gene involved in the utilization of acetate is subject to CCR $[10,28,29]$. Consistently, acetate was secreted as soon as glucose was imported and metabolized by both strains. When the stationary phase was reached, acetate uptake was not noticed, as commonly reported [11,30]. Although acetate could be reimported and utilized as an alternative carbon source after the depletion of glucose (and the consequent diminish of the CCR effect), cells preferred to utilize pyruvate. Thus, acetate efflux continued until the end of cultivation. Yet, it is speculated that acetate influx could be observed if cells were cultivated for longer. Furthermore, it is also hypothesized, that acetate uptake could have been subject of catabolic repression by pyruvate since the latter was consumed as an alternative carbon source during the exponential phase.

Acetoin and 2,3-butanediol secretions were also detected. Notably, the effluxes were much higher in $\Delta$ pyk than in wt. The reason for these differences remains unclear. The upper high efflux of acetoin was previously observed [31]. The production of these overflow metabolites is perceived as a preventive mechanism of environment acidification due to acetate accumulation [29,32]. Acetoin is synthesized from pyruvate and can either be secreted or converted into 2,3-butanediol in order to generate $\mathrm{NAD}^{+}$. These compounds can also be reimported during the stationary phase and, in this way, are used as an energy-storing strategy. The fact that $\Delta$ pyk secreted more acetoin and 2,3-butanediol than in wt leads us to think that the perturbation occurring in the pyruvate node could result in the imbalance of the NADH/NAD ${ }^{+}$ratio, which is known to be potentially toxic to the cells $[26,33]$. Consistently, the higher synthesis of acetoin and subsequent 2,3-butanediol results in more $\mathrm{NAD}^{+}$molecules being regenerated, which can help balance the reducing power ratio. Likewise, the secretion of acetoin and, to an extent, 2,3-butanediol occurred alongside acetate accumulation, which is in agreement with the fact that acetoin synthesis can also be induced by acetate $[32,34,35]$. On the contrary to acetate, acetoin and 2,3-butanediol uptake was observed when the stationary phase was reached, suggesting that they were used as alternative carbon sources as soon as glucose and pyruvate were exhausted.

For a robust statistical analysis, these studies were performed using four biological replicates, instead of the common use of triplicates. Nevertheless, statistical corrections could have been applied, such as Bonferroni or Sidak methods, to increase the power of the statistical outcomes.

These findings may shed light on the mechanisms of B. subtilis to regulate the transport of glucose and pyruvate and the possible metabolic reroute to alternative pathways in the absence of Pyk.

\section{Materials and Methods}

\subsection{Bacterial Strains and Growth Conditions}

Cloning and genetic manipulations were conducted using standard procedures [36,37]. B. subtilis strains were derived from BSB1, a tryptophane $\left(\operatorname{trp}^{+}\right)$derivative of $B$. subtilis 168 . The deletion of the pyk gene was performed in the Department of General Microbiology, Georg-August-Universität Göttingen with the long flanking homology PCR technique, as previously described by Pietack et al. [38,39]. This was achieved by transformation with PCR products constructed using oligonucleotides to amplify DNA fragments flanking the target genes and an intervening antibiotic resistance cassette. For that purpose, genes that mediate resistance against erythromycin were amplified from the plasmid pDG646 [40]. The PCR product was purified using the QIAquick PCR Purification Kit (Qiagen; Hilden; Germany). B. subtilis was transformed with the purified PCR products and transformants were selected 
on plates with the antibiotic. Clones were examined by checking PCR for the integrity of the resistance cassette. The DNA sequence of the flanking regions was verified by sequencing. Both B. subtilis BSB1 wild-type (wt) and pyruvate kinase mutant ( $\Delta$ pyk) were grown in Lysogeny Broth (LB) and M9 media was prepared as previously described by Harward and Cutting with small changes [36]. To prevent precipitation problems, magnesium sulfate $(1 \mathrm{M})$ and calcium chloride $(100 \mathrm{mM})$ were added as separated solutions. Moreover, iron chloride solution (50 mM) was prepared with citric acid $(100 \mathrm{mM})$ and was also added as a separate solution. For growth experiments, different carbon sources were added to the M9 stock medium: (i) glucose $(20 \mathrm{mM})$ and malate (5 mM) (M9GlcMal); (ii) glucose $(10 \mathrm{mM})$ and pyruvate $(60 \mathrm{mM})(\mathrm{M} 9 \mathrm{GlcPyr})$.

\subsection{Cultivation}

LB agar plates of $B$. subtilis were prepared from frozen stocks $\left(-80{ }^{\circ} \mathrm{C}\right.$, in $15 \%(v / v)$ glycerol) and incubated overnight at $37^{\circ} \mathrm{C}$. For genetic selection, kanamycin $(3 \mu \mathrm{g} / \mathrm{mL})$ was added to the mutant plates.

The pre-cultures were incubated from isolated colonies for $4 \mathrm{~h}$ in $5 \mathrm{~mL}$ of LB medium at $37^{\circ} \mathrm{C}$ and $300 \mathrm{rpm}$. These cultivations were used to prepare a second set of overnight cultures in M9GlcMal in several dilutions to ensure it was in the exponential growth phase. After $15 \mathrm{~h}$ of incubation at $37^{\circ} \mathrm{C}$ and $260 \mathrm{rpm}$, the cultures in exponential growth-optical density $\left(\mathrm{OD}_{600 \mathrm{~nm}}\right)$ between $0.5-0.8$ - were centrifuged at $6000 \mathrm{rpm}$ and $4{ }^{\circ} \mathrm{C}$ for $3 \mathrm{~min}$ (Heraeus Multifuge X1R, Thermo Scientific, Waltham, MA, USA). The cells were resuspended in the main culture medium and were inoculated with an initial $\mathrm{OD}_{600 \mathrm{~nm}}$ of 0.1 at $37^{\circ} \mathrm{C}$ and $300 \mathrm{rpm}$.

\subsection{Sampling of Extracellular and Intracellular Metabolites}

To investigate the extracellular metabolite composition, $2 \mathrm{~mL}$ of bacterial culture was sterile filtered $\left(0.45 \mu \mathrm{m}\right.$ pore size, Filtropur S, Sarstedt AG) every $60 \mathrm{~min}$ and stored at $-20{ }^{\circ} \mathrm{C}$ prior to ${ }^{1} \mathrm{H}-\mathrm{NMR}$ measurements.

For intracellular metabolite samples, 20 OD units of cell culture were harvested via a vacuum-dependent fast-filtration system as described by Meyer et al. with few modifications (Figure S1) [41].

In brief, the main culture was transferred into a falcon tube and cooled by dipping the sample periodically in liquid nitrogen for $10 \mathrm{~s}$ maximum (approximately $1 \mathrm{~s}$ each time). During this in/out of liquid nitrogen cycle, the sample was carefully shaken to avoid freezing and metabolite leakage caused by cell lysis. Subsequently, the cooled cell culture was filtered (regenerated cellulose membrane filter, $0.45 \mu \mathrm{m}$ pore size, $100 \mathrm{~mm}$ diameter, RC55 Whatman) and washed 2 times with isotonic sodium chloride solution at $4{ }^{\circ} \mathrm{C}(0.8$ or $0.9 \%$ when cultivated in chemically defined or complex medium, respectively). The filter was immediately transferred to a falcon tube containing $5 \mathrm{~mL}$ of ice-cold extraction solution ( $60 \% w / v$ of ethanol absolute $99.8 \%$ ) and the internal standard (ISTD) constituted of $2.5 \mathrm{nmol}$ of camphor sulfonic acid (CSA) for HPLC-MS, $20 \mathrm{nmol}$ of labeled amino acids (Cell Free Amino Acid Mixture- $-{ }^{13} \mathrm{C},{ }^{15} \mathrm{~N}$; Sigma Aldrich, St. Louis, MO, USA) for LC-MS/MS, and 20 nmol of ribitol for GC-MS analysis. The metabolites were quenched by freezing the sample immediately in liquid nitrogen. The falcon tube was stored at $-80{ }^{\circ} \mathrm{C}$ until extraction. Subsequently, the sample was snap-frozen in liquid nitrogen. The sampling procedure took less than $1 \mathrm{~min}$. The efficient quenching of the metabolism was confirmed with the determination of the adenylate energy charge (AEC) of each biological sample, according to the method of Atkinson [42]. In both strains, the AEC was over 0.7 (data not shown).

The sample was stored at $-80{ }^{\circ} \mathrm{C}$ until extraction. For cell disruption and metabolites extraction, 10 freeze/thaw cycles were performed by alternately thawing it on ice, vortexing and shaking the samples. Afterwards, the sample was centrifuged for $5 \mathrm{~min}$ at $4{ }^{\circ} \mathrm{C}$ and $13,000 \mathrm{rpm}$. The supernatant was collected in a new falcon tube and left on ice. The pellet formed was extracted once again with $5 \mathrm{~mL}$ of water (HPLC-MS grade). The two supernatants were combined and restocked with distilled 
water to a final organic solution concentration of $10 \%$ and stored at $-80{ }^{\circ} \mathrm{C}$ prior to lyophilization. The sample was lyophilized with a Christ Alpha 1-4 LSC lyophilizer at $-52{ }^{\circ} \mathrm{C}$ and 0.25 mbar. To avoid disturbances in HPLC columns associated with the accumulation of macromolecules (i.e., proteins), a third extraction was added to the experimental procedure. Therefore, $500 \mu \mathrm{L}$ of cold water (HPLC-MS grade) and $100 \mu \mathrm{L}$ of cold chloroform (HPLC-MS grade) were added to the sample, followed by $1 \mathrm{~min}$ of periodically shaking and vortexing. Afterwards, sample was stored at $-20{ }^{\circ} \mathrm{C}$ for $10 \mathrm{~min}$. The supernatant was separated by centrifugation for $10 \mathrm{~min}$ at $4{ }^{\circ} \mathrm{C}$ and $13,000 \mathrm{rpm}$. The aqueous phase was collected and lyophilized once again. The sample was stored at $-20{ }^{\circ} \mathrm{C}$ until analytical analyses.

\subsection{H-NMR Spectroscopy Measurement and Data Analysis of Extracellular Metabolites}

A volume of $400 \mu \mathrm{L}$ of sample was mixed with $200 \mu \mathrm{L}$ of sodium hydrogen phosphate buffer (0.2 mM, pH 7.0 made up with 50\% $\mathrm{D}_{2} \mathrm{O}$ ) containing 3-trimethylsilyl-[2,2,3,3- $\left.\mathrm{D}_{4}\right]$-1-propionic acid $(1 \mathrm{mM})$ to provide a nuclear magnetic resonance NMR-lock signal. All NMR spectra were obtained at $600.27 \mathrm{MHz}$ at $310 \mathrm{~K}$ using a Bruker Avance-II $600 \mathrm{NMR}$ spectrometer operated by TOPSPIN 3.2 software (Bruker Biospin GmbH, Rheinstetten, Germany). A modified 1D-1 H-nuclear Overhauser effect spectroscopy (1D-NOESY) pulse sequence was used with presaturation on the residual peak (HDO) during both relaxation delay and mixing time. A total of 64 free induction decays (FID scans) were collected, using a spectral width of 30 ppm for a one-dimensional spectrum.

The identification and quantification analysis were carried out using AMIX v3.9.11 software (Bruker Biospin GmbH, Rheinstetten, Germany). The signal peak identification was based on spectra alignment of pure standard compounds (Sigma-Aldrich, St. Louis, MO, USA). Quantification was carried out by integration and comparison of designated peaks to an external standardized computed $10 \mathrm{mM}$ signal at $15 \mathrm{ppm}$. Unidentified signals were relatively quantified due to their unknown quantity of protons.

\subsection{GC-MS Measurement and Data Analysis of Intracellular Metabolites}

The dried samples were derivatized firstly with $60 \mu \mathrm{L}$ of methoxyamine $(20 \mathrm{mg} / \mathrm{mL}$ pyridine $)$ for $90 \mathrm{~min}$ at $37{ }^{\circ} \mathrm{C}$ and secondly with $120 \mu \mathrm{L}$ of $N$-methyl- $N$-trimethylsilyltrifluroacetamide (Chromatographie-Service $\mathrm{GmbH}$ ) for $30 \mathrm{~min}$ at $37{ }^{\circ} \mathrm{C}$. Samples were centrifuged for $2 \mathrm{~min}$ at room temperature and the supernatant was transferred into GC-vial for injection. GC-MS analysis was performed with an Agilent 6890N GC system with an auto-sampler G2614A model coupled to a mass selective detector 5973N model (Agilent Technologies, St. Clara, CA, USA). A $2 \mu L$ sample was injected (G2613A model Series Injector) with a split 1:10 at $250{ }^{\circ} \mathrm{C}$, using helium as the carrier gas (split flow of $10 \mathrm{~mL} \mathrm{~min}^{-1}$ and $8.8 \mathrm{Psi}$ ).

The chromatographic run was performed as described by Dörries et al. [43]. Using a $30 \mathrm{~m} \mathrm{DB}$ 5-column (JW Scientific, Folsom, CA, USA) with $0.25 \mathrm{~mm}$ inner diameter and $2.5 \mu \mathrm{m}$ film thickness, and a constant gas flow of $1 \mathrm{~mL} / \mathrm{min} \mathrm{HPr}-\mathrm{Ser}-\mathrm{P}-\mathrm{CcpA}$ complex. The oven program started with an initial temperature hold at $70{ }^{\circ} \mathrm{C}$ for $1 \mathrm{~min}$ and continued with a heating rate of $1{ }^{\circ} \mathrm{C} \min ^{-1}$ up to $76^{\circ} \mathrm{C}$, $5{ }^{\circ} \mathrm{C} \mathrm{min}$ min $^{-1}$ up to $220^{\circ} \mathrm{C}$, and $20^{\circ} \mathrm{C} \mathrm{min}^{-1}$ up to $330^{\circ} \mathrm{C}$, with a hold for $3 \mathrm{~min}$ followed by a $10 \mathrm{~min}$ isothermal cool-down to $70^{\circ} \mathrm{C}$. The analytes were transferred to a quadrupole mass analyzer operated in the electron impact ionization (EI) mode with an ionization energy of $70 \mathrm{eV}$. Data acquisition was carried out in 40 min runtime. Full scan mass spectra were acquired from 50 to $500 \mathrm{~m} / \mathrm{z}$ at a rate of 2 scans/sec and with a 6 min solvent delay.

The qualitative analysis of the detected compounds was performed using ChromaTOF software (LECO Corporation, St. Joseph, MI, USA). Metabolite identification was carried out by comparison of retention time and fragmentation patterns peaks detected to the NIST mass spectral database 2.0 (Gaithersburg, MD, USA) and an in-house database. For relative quantification, the area of the quantifier ion of each metabolite was integrated and normalized to the area of the ISTD (ribitol). 
For precision analysis control, daily quality control (dQC) samples were analyzed during the batch. The dQC consisted of 53 metabolites with $100 \mathrm{nmol}$ concentration for each metabolite. Precision analysis was determined by assessing the measured dQC in calibration curves with concentrations ranging from $0.5 \mathrm{nmol}$ to $500 \mathrm{nmol}$ of each metabolite. The calibration curves were fit with a polynomial of degree 2 and 1/x weighting based on minimum of 6 calibration points.

\subsection{HPLC-MS Measurement and Data Analysis of Intracellular Metabolites}

For the intracellular metabolite analysis in HPLC-MS, the lyophilized samples were dissolved in $100 \mu \mathrm{L}$ of water (HPLC-MS grade) and centrifuged for $2 \mathrm{~min}$ at room temperature. The supernatant was transferred into a HPLC vial for injection. The HPLC-MS analysis was carried out using an Agilent 1100 HPLC system consisted of a degasser, a quaternary pump and a G1329A autosampler with controlled temperature coupled to Bruker micro-TOF mass spectrometer (Bruker Daltonics, Bremen, Germany).

Chromatography was performed on a SymmetryShield RP18 column $(3.5 \mu \mathrm{m}, 150 \times 4.6 \mathrm{~mm})$ (Waters, Milford, MA, USA) with a SecurityGuard cartridge C18 pre-column $(4 \times 3.0 \mathrm{~mm})$ (Phenomenex, Torrance, CA, USA) using an ion-pairing reagent and a methanol gradient as described by Dörries et al. [43]. In detail, the mobile phase consisted of eluent A: $95 \%$ water and $5 \%$ methanol, containing $10 \mathrm{mM}$ of tributylamine as the ion-pairing reagent and $15 \mathrm{mM}$ of acetic acid, $\mathrm{pH} 4.9$; and eluent B: $100 \%$ methanol. Data acquisition was carried out in $42 \mathrm{~min}$ runtime with a flow rate of $0.4 \mathrm{~mL} \mathrm{~min}^{-1}$. The gradient elution started with $100 \% \mathrm{~A}$ for $2 \mathrm{~min}, 0-31 \% \mathrm{~B}$ in $2 \mathrm{~min}$ and continued with 31 to $50 \%$ in $18 \mathrm{~min}$. Followed by $50-60 \%$ B in $2 \mathrm{~min}, 60-100 \%$ B in $1 \mathrm{~min}$ and left $100 \%$ for $7 \mathrm{~min}$. The eluent $A$ returned to $100 \%$ in $1 \mathrm{~min}$ and was left for $10 \mathrm{~min}$ until the end of the run. The gradient elution started with $100 \%$ A for $2 \mathrm{~min}, 0-31 \%$ B in $2 \mathrm{~min}$ and continued with 31 to $50 \%$ in $18 \mathrm{~min}$. Followed by $50-60 \%$ B in $2 \mathrm{~min}, 60-100 \%$ B in $1 \mathrm{~min}$ and left $100 \%$ for $7 \mathrm{~min}$. The eluent A returned to $100 \%$ in $1 \mathrm{~min}$ and was left for $10 \mathrm{~min}$ until the end of the run.

Mass spectrometry was operated in electrospray ionization and negative-ion mode using a mass scan range of 50 to $3000 \mathrm{~m} / \mathrm{z}$. Internal MS calibration was carry out in the beginning of each chromatographic run with 16 different masses from a sodium formate solution tune mix ( $49.4 \%$ water, $49.4 \%$ isopropanol, $0.2 \%$ formic acid, and $10 \mathrm{mM}$ sodium hydroxide).

Metabolite identification was carried out by comparison of retention time and $\mathrm{m} / \mathrm{z}$ values of detected peaks $\left([\mathrm{M}-\mathrm{H}]^{-}\right.$or $\left.[\mathrm{M}-2 \mathrm{H}]^{2-}\right)$ with database alignment of the calculated exact mass.

The quantitative analysis was carried out using QuantAnalysis (Bruker Daltonik, Bremen, Germany). The extracted ion peaks were integrated and normalized to the ISTD (CSA) area.

The dQC samples consisted of 22 metabolites with $10 \mathrm{nmol}$ concentration of each metabolite. Precision analysis was determined by assessing the measured dQC in calibration curves with concentrations ranging from $0.5 \mathrm{nmol}$ to $500 \mathrm{nmol}$ of each metabolite. The calibration curves fitting was performed with a polynomial of degree 2 and $1 / x$ weighting based on a minimum of 6 calibration points.

\subsection{LC-MS/MS Measurement and Data Analysis of Intracellular Metabolites}

The amino acids proline, arginine, and citrulline were analyzed without derivatization on an Intrada amino acid column $(50 \times 3 \mathrm{~mm}, 3 \mu \mathrm{m})$ (Imtakt Corporation) with acetonitrile/100 mM ammonium-formate $(20 / 80, v / v)$ as eluent $\mathrm{A}$ and acetonitrile/THF/25 mM ammonium formate/formic acid $(9 / 75 / 16 / 0.3, v / v / v / v)$ as eluent $B$.

A $2 \mu \mathrm{L}$ sample was injected in the column stabilized at $40^{\circ} \mathrm{C}$. Data acquisition was carried out in 26 min runtime with a flow rate of $0.4 \mathrm{~mL} \mathrm{~min}^{-1}$.

The gradient elution started with $100 \%$ B for $5 \mathrm{~min}$, followed by $0-17 \%$ A in $4.45 \mathrm{~min}$. Eluent A reached $100 \%$ in $1.15 \mathrm{~min}$ and was left in this condition for $6 \mathrm{~min}$. Afterwards, eluent B returned to $100 \%$ in 50 sec and was left for 8 min until the end of the run. 
Analyses were performed on a HPLC system (1200) consisting of a degasser, a binary pump, a temperature-controlled autosampler, and a column oven coupled to a 6460 triple quadrupole mass spectrometer with Electrospray ionization (ESI) Jet stream source (all Agilent Technologies). The mass spectrometer was operated in the positive mode (Table S4) using multiple reaction monitoring (MRM).

Proline $-{ }^{13} \mathrm{C},{ }^{15} \mathrm{~N}$ and arginine $-{ }^{13} \mathrm{C},{ }^{15} \mathrm{~N}$ were used as internal standard compounds for relative quantification (Cell Free Amino Acid Mixture- $-{ }^{13} \mathrm{C},{ }^{15} \mathrm{~N}$; Sigma Aldrich).

Quantification was carried out using Mass Hunter Quantitative Analysis (for QQQ) B 06.00 from Agilent.

\subsection{Statistical Analysis and Visualization}

Microsoft Excel software 2007 was used for metabolite quantification and calculation of standard deviations (SD) and fold changes (FCs).

The visualization of the time-resolved extracellular metabolite changes was performed using Excel and VANTED software v2.01.

The heat map of metabolites was generated using MeV v4.8.1 with hierarchical clustering analysis using Euclidean distance and average linkage method.

Bar charts, XY plots were created using GraphPad PRISM software v6.01. Unpaired t-tests were also carried out in GraphPad PRISM. The two-sided homoscedastic $t$-tests were used to calculate $p$-values, whereas $p$-values $\leq 0.05$ were considered as being statistically significant.

The area of $\mathrm{m} / \mathrm{z}$ detected for each metabolite in the spectrometric techniques was integrated and normalized to the integral of the area of $m / z$ the internal standard by using, resulting in the relative metabolite amount per 20 OD units.

The metabolite missing values were replaced with half the minimum positive value in the original data.

\section{Conclusions}

This study shows the metabolic responses of B. subtilis lacking Pyk. We were able to highlight relevant metabolic differences between wt and $\Delta$ pyk cells when monitored in a chemically defined medium with glucose and pyruvate as carbon sources.

In this work, a new quality of information regarding the metabolism and adaptation to the absence of key signal mechanisms in B. subtilis was provided. Investigations of cells lacking Pyk uncovered alterations in the import of glucose and pyruvate from the nutritional media. The concomitant consumption of both metabolites in $\Delta$ pyk cells was observed, whereas in wt, the uptake of pyruvate was perceived after the complete consumption of glucose. This distinct behavior is in accordance with the pyruvate transport mechanism recently discovered in B. subtilis. While in wt, the pyruvate utilization suffers CCR by the CcpA-dependent glucose repressor, in $\Delta$ pyk, this effect is possibly relieved due to the induction of the pyruvate facilitated transporter genes, by LytST. The lack of Pyk and consequent low intracellular pyruvate level could be involved in this induction, since LytST works in a pyruvate dose- and gradient-manner. Although the metabolic results provided help to elucidate the regulatory transport of glucose and pyruvate, complete knowledge of the pyruvate transport mechanism and the adaptation to new gradient concentrations by B. subtilis is yet to be determined. Other prominent pathways were affected by the pyk mutation such as the overflow metabolism, TCA cycle, and the PPP. Considering that pyruvate is a link of essential pathways and its fate is important for cell robustness and viability, the metabolic approach of this study helps to unravel the control of pyruvate homeostasis and B. subtilis adaptation to environmental challenges. 
Supplementary Materials: The following are available online at http://www.mdpi.com/2218-1989/9/10/216/s1, Table S1: OD of wt and $\Delta$ pyk at $600 \mathrm{~nm}$ during growth in M9GlcPyr at $37^{\circ} \mathrm{C}$ and $300 \mathrm{rpm}$, Table S2: Physiological parameters (growth rate, acetate, acetoin, and 2,3-butanediol maximum concentration) of wt and $\Delta$ pyk of four replicates, Table S3: Intracellular metabolome data of B. subtilis wt and $\Delta$ pyk. Mean of the relative amount of intracellular metabolites, SD values, and FC of four biological replicates of wt and $\Delta$ pyk, Unpaired $t$-tests were also determined for each metabolite, where $p$-value represents the statistical result, Figure S1: Experimental workflow for metabolome analysis, Table S4: MS source parameters used during amino acids measurements.

Author Contributions: J.S., P.W., and M.L. conceived and designed the experiments, J.S. and K.M. performed the experiments; J.S., K.M., P.W. analyzed the data and prepared the original draft; all the authors reviewed and edited the manuscript.

Funding: This research was funded by the European Union, Marie Curie ITN AMBER, grant number 317338.

Acknowledgments: We are grateful to Jörg Stülke and Nora Cascante-Estepa for providing the strains. We thank Hanna Meyer and Kirsten Dörries for the technical assistance and Baltic-Analytics for sharing laboratory facilities.

Conflicts of Interest: The authors declare no conflict of interest.

\section{References}

1. Kunst, F.; Ogasawara, N.; Moszer, I.; Albertini, A.M.; Alloni, G.O.; Azevedo, V.; Bertero, M.G.; Bessieres, P.; Bolotin, A.; Borchert, S.; et al. The complete genome sequence of the gram-positive bacterium Bacillus subtilis. Nature 1997, 390, 249-256. [CrossRef]

2. Barbe, V.; Cruveiller, S.; Kunst, F.; Lenoble, P.; Meurice, G.; Sekowska, A.; Vallenet, D.; Wang, T.; Moszer, I.; Médigue, C.; et al. From a consortium sequence to a unified sequence: The Bacillus subtilis 168 reference genome a decade later. Microbiology 2009, 155, 1758-1775. [CrossRef]

3. Schallmey, M.; Singh, A.; Ward, O.P. Developments in the use of Bacillus species for industrial production. Can. J. Microbiol. 2004, 50, 1-17. [CrossRef]

4. Zhang, A.; Sun, H.; Wang, P.; Han, Y.; Wang, X. Modern analytical techniques in metabolomics analysis. Analyst 2012, 137, 293-300. [CrossRef] [PubMed]

5. Sauer, U.; Eikmanns, B.J. The PEP-pyruvate-oxaloacetate node as the switch point for carbon flux distribution in bacteria: We dedicate this paper to Rudolf K. Thauer, Director of the Max-Planck-Institute for Terrestrial Microbiology in Marburg, Germany, on the occasion of his 65th birthday. FEMS Microbiol. Rev. 2005, 29, 765-794. [PubMed]

6. Goel, A.; Lee, J.; Domach, M.M.; Ataai, M.M. Metabolic fluxes, pools, and enzyme measurements suggest a tighter coupling of energetics and biosynthetic reactions associated with reduced pyruvate kinase flux. Biotechnol. Bioeng. 1999, 64, 129-134. [CrossRef]

7. Pan, Z.; Zhu, T.; Domagalski, N.; Khan, S.; Koepsel, R.R.; Domach, M.M.; Ataai, M.M. Regulating expression of pyruvate kinase in Bacillus subtilis for control of growth rate and formation of acidic byproducts. Biotechnol. Prog. 2006, 22, 1451-1455. [CrossRef] [PubMed]

8. Charbonnier, T.; Le Coq, D.; McGovern, S.; Calabre, M.; Delumeau, O.; Aymerich, S.; Jules, M. Molecular and Physiological Logics of the Pyruvate-Induced Response of a Novel Transporter in Bacillus subtilis. MBio 2017, 8, e00976-17. [CrossRef] [PubMed]

9. Albanesi, D.; De Mendoza, D. FapR: From Control of Membrane Lipid Homeostasis to a Biotechnological Tool. Front. Mol. Biosci. 2016, 3, 64. [CrossRef]

10. Fujita, Y. Carbon catabolite control of the metabolic network in Bacillus subtilis. Biosci. Biotechnol. Biochem. 2009, 73, 245-259. [CrossRef]

11. Görke, B.; Stülke, J. Carbon catabolite repression in bacteria: Many ways to make the most out of nutrients. Nat. Rev. Microbiol. 2008, 6, 613-624. [CrossRef] [PubMed]

12. Kleijn, R.J.; Buescher, J.M.; Le Chat, L.; Jules, M.; Aymerich, S.; Sauer, U. Metabolic fluxes during strong carbon catabolite repression by malate in Bacillus subtilis. J. Biol. Chem. 2010, 285, 1587-1596. [CrossRef] [PubMed]

13. Singh, K.D.; Schmalisch, M.H.; Stülke, J.; Görke, B. Carbon catabolite repression in Bacillus subtilis: Quantitative analysis of repression exerted by different carbon sources. J. Bacteriol. 2008, 190, 7275-7284. [CrossRef] [PubMed] 
14. van den Esker, M.H.; Kovács, Á.T.; Kuipers, O.P. YsbA and LytST are essential for pyruvate utilization in Bacillus subtilis. Environ. Microbiol. 2017, 19, 83-94. [CrossRef] [PubMed]

15. van den Esker, M.H.; Kovács, Á.T.; Kuipers, O.P. From Cell Death to Metabolism: Holin-Antiholin Homologues with New Functions. MBio 2017, 8, e01963-17. [CrossRef] [PubMed]

16. Deutscher, J.; Francke, C.; Postma, P.W. How phosphotransferase system-related protein phosphorylation regulates carbohydrate metabolism in bacteria. Microbiol. Mol. Biol. Rev. 2006, 70, 939-1031. [CrossRef] [PubMed]

17. Postma, P.; Lengeler, J.; Jacobson, G. Phosphoenolpyruvate: Carbohydrate phosphotransferase systems of bacteria. Microbiol. Rev. 1993, 57, 543-594.

18. Li, Z.; Nimtz, M.; Rinas, U. The metabolic potential of Escherichia coli BL21 in defined and rich medium. Microb. Cell Fact. 2014, 13, 45. [CrossRef]

19. Sonenshein, A.L. Control of key metabolic intersections in Bacillus subtilis. Nat. Rev. Microbiol. 2007, 5, 917-927. [CrossRef]

20. Ponce, E.; Martínez, A.; Bolívar, F.; Valle, F. Stimulation of glucose catabolism through the pentose pathway by the absence of the two pyruvate kinase isoenzymes in Escherichia coli. Biotechnol. Bioeng. 1998, 58, 292-295. [CrossRef]

21. Emmerling, M.; Dauner, M.; Ponti, A.; Fiaux, J.; Hochuli, M.; Szyperski, T.; Wüthrich, K.; Bailey, J.E.; Sauer, U. Metabolic flux responses to pyruvate kinase knockout in Escherichia coli. J. Bacteriol. 2002, 184, 152-164. [CrossRef] [PubMed]

22. Meza, E.; Becker, J.; Bolivar, F.; Gosset, G.; Wittmann, C. Consequences of phosphoenolpyruvate: Sugar phosphotranferase system and pyruvate kinase isozymes inactivation in central carbon metabolism flux distribution in Escherichia coli. Microb. Cell Fact. 2012, 11, 127. [CrossRef] [PubMed]

23. Toya, Y.; Ishii, N.; Nakahigashi, K.; Hirasawa, T.; Soga, T.; Tomita, M.; Shimizu, K. 13C-metabolic flux analysis for batch culture of Escherichia coli and its pyk and pgi gene knockout mutants based on mass isotopomer distribution of intracellular metabolites. Biotechnol. Prog. 2010, 26, 975-992. [PubMed]

24. Schilling, O.; Frick, O.; Herzberg, C.; Ehrenreich, A.; Heinzle, E.; Wittmann, C.; Stülke, J. Transcriptional and metabolic responses of Bacillus subtilis to the availability of organic acids: Transcription regulation is important but not sufficient to account for metabolic adaptation. Appl. Environ. Microbiol. 2007, 73, 499-507. [CrossRef]

25. Dauner, M.; Storni, T.; Sauer, U. Bacillus subtilis metabolism and energetics in carbon-limited and excess-carbon chemostat culture. J. Bacteriol. 2001, 183, 7308-7317. [CrossRef]

26. Vemuri, G.N.; Altman, E.; Sangurdekar, D.P.; Khodursky, A.B.; Eiteman, M.A. Overflow metabolism in Escherichia coli during steady-state growth: Transcriptional regulation and effect of the redox ratio. Appl. Environ. Microbiol. 2006, 72, 3653-3661. [CrossRef]

27. Szenk, M.; Dill, K.A.; de Graff, A.M.R. Why do fast-growing bacteria enter overflow metabolism? Testing the membrane real estate hypothesis. Cell Syst. 2017, 5, 95-104. [CrossRef]

28. Wolfe, A.J. The acetate switch. Microbiol. Mol. Biol. Rev. 2005, 69, 12-50. [CrossRef]

29. Grundy, F.; Waters, D.; Allen, S.; Henkin, T. Regulation of the Bacillus subtilis acetate kinase gene by CcpA. J. Bacteriol. 1993, 175, 7348-7355. [CrossRef]

30. Meyer, H.; Weidmann, H.; Mäder, U.; Hecker, M.; Völker, U.; Lalk, M. A time resolved metabolomics study: The influence of different carbon sources during growth and starvation of Bacillus subtilis. Mol. Biosyst. 2014, 10, 1812-1823. [CrossRef]

31. Cabrera-Valladares, N.; Martínez, L.M.; Flores, N.; Hernández-Chávez, G.; Martínez, A.; Bolívar, F.; Gosset, G. Physiologic consequences of glucose transport and phosphoenolpyruvate node modifications in Bacillus subtilis 168. J. Mol. Microbiol. Biotechnol. 2012, 22, 177-197. [CrossRef] [PubMed]

32. Xiao, Z.; Xu, P. Acetoin metabolism in bacteria. Crit. Rev. Microbiol. 2007, 33, 127-140. [CrossRef] [PubMed]

33. Ramos, H.C.; Hoffmann, T.; Marino, M.; Nedjari, H.; Presecan-Siedel, E.; Dreesen, O.; Glaser, P.; Jahn, D. Fermentative metabolism of Bacillus subtilis: Physiology and regulation of gene expression. J. Bacteriol. 2000, 182, 3072-3080. [CrossRef] [PubMed] 
34. Turinsky, A.J.; Grundy, F.J.; Kim, J.-H.; Chambliss, G.H.; Henkin, T.M. Transcriptional activation of the Bacillus subtilis ackA gene requires sequences upstream of the promoter. J. Bacteriol. 1998, 180, 5961-5967.

35. Härtig, E.; Jahn, D. Regulation of the anaerobic metabolism in Bacillus subtilis. Adv. Microb. Physiol. 2012, $61,195-216$.

36. Harwood, C.R.; Cutting, S.M. Molecular Biological Methods for Bacillus; Wiley: Hoboken, NJ, USA, 1990.

37. Sambrook, J. Molecular Cloning: A Laboratory Manual, 3rd ed.; Cold Spring Harbor Laboratory Press: Long Island, NY, USA, 2001.

38. Wach, A. PCR-synthesis of marker cassettes with long flanking homology regions for gene disruptions in $S$. cerevisiae. Yeast 1996, 12, 259-265. [CrossRef]

39. Pietak, N.; Becher, D.; Schmidl, S.R.; Saier, M.H.; Hecker, M.; Commichau, F.M.; Stülke, J. In vitro Phosphorylation of Key Metabolic Enzymes from Bacillus subtilis: PrkC Phosphorylates Enzymes from Different Branches of Basic Metabolism. J. Mol. Microbiol. Biotechnol. 2010, 18, 129-140. [CrossRef]

40. Guerout-Fleury, A.-M.; Shazand, K.; Frandsen, N.; Stragier, P. Antibiotic-resistance cassettes for Bacillus subtilis. Genes 1995, 167, 335-336. [CrossRef]

41. Meyer, H.; Weidmann, H.; Lalk, M. Methodological approaches to help unravel the intracellular metabolome of Bacillus subtilis. Microb. Cell Fact. 2013, 12, 69. [CrossRef]

42. Atkinson, D.E. Energy charge of the adenylate pool as a regulatory parameter. Interaction with feedback modifiers. Biochemistry 1968, 7, 4030-4034. [CrossRef]

43. Dörries, K.; Schlueter, R.; Lalk, M. Impact of antibiotics with various target sites on the metabolome of Staphylococcus aureus. Antimicrob. Agents Chemother. 2014, 58, 7151-7163. [CrossRef] [PubMed]

(C) 2019 by the authors. Licensee MDPI, Basel, Switzerland. This article is an open access article distributed under the terms and conditions of the Creative Commons Attribution (CC BY) license (http://creativecommons.org/licenses/by/4.0/). 